\title{
Highly Sodium-Selective Fluoroionophore Based on Conformational Restriction of Oligoethyleneglycol-bridged Biaryl Boron-dipyrromethene
}

\author{
Koji Yamada, Yuki Nomura, Daniel Citterio, Naoko Iwasawa, and Koji Suzuki \\ Contribution from Department of Applied Chemistry, Faculty of Science and Technology, Keio \\ University, 3-14-1 Hiyoshi, Hokoku-ku, Yokohama 223-8522, Japan, Kanagawa Academy of Science \\ and Technology (KAST), KSP West 614, 3-2-1 Sakado, Takatsu-ku, Kawasaki 213-0012, and Core \\ Research for Evolutional Science and Technology (CREST), JST Agency, 4-1-8 Honcho, Kawaguchi,
}

Saitama 332-0012, Japan

\section{Supporting Information}

Most of the chemicals were of reagent grade quality obtained from commercial sources and used without further purification. The others were prepared by the procedure given in the reference. The NMR spectra were recorded using a JNM-LA300 (JEOL) instrument. The MS spectrum of 1-Na ${ }^{+}$was obtained using a Mariner ESI-TOF mass spectrometer (PerSeptive Biosystems). The absorption and emission spectra were measured using a U-2001 spectrometer (Hitachi) and a F-4500 fluorescence spectrophotometer (Hitachi). The binding constant $(K)$ of $\mathbf{1}$ with the alkali ions was calculated using the Benesi-Hildebrand equation as follows: ${ }^{1}$

$[1] / \Delta A=1 / K \Delta A_{\max } \times 1 /[M]+1 / \Delta A_{\max }$

where $[1]$ is the total concentration of $\mathbf{1}, \Delta A$ is the difference in the absorbance, and $[M]$ is the concentration of the added alkali ion. From the linear plots of $[M]$ vs. $[1] / \Delta A, K$ is determined from the slope and the y-intercept. N, N'-Bis(1-hexylheptyl)-3,4:9,10-perylenebis(dicarboximide) in $\mathrm{CH}_{2} \mathrm{Cl}_{2}$ was used as a fluorescence reference $\left(\Phi_{\mathrm{f}}=1.00\right){ }^{2}$ The fluorescence quantum yield was obtained using the following equation: ${ }^{3}$

$\Phi_{s}=\left[\left(A_{r} F_{s} n_{s}^{2}\right) /\left(A_{s} F_{r} n_{r}^{2}\right)\right] \Phi_{r}$

where the $s$ subscript refers to the fluoroionophore 1 and $r$ to the reference. The other symbols have the following meanings: $\Phi$ is the fluorescence quantum yield, $A$ is absorbance at the excitation wavelength, $F$ is the integrated emission area across the band and $n_{s}$ and $n_{r}$ are the refractive index of acetonitrile and $\mathrm{CH}_{2} \mathrm{Cl}_{2}$, respectively. 


\section{Experimental Procedures and Spectroscopic Data}
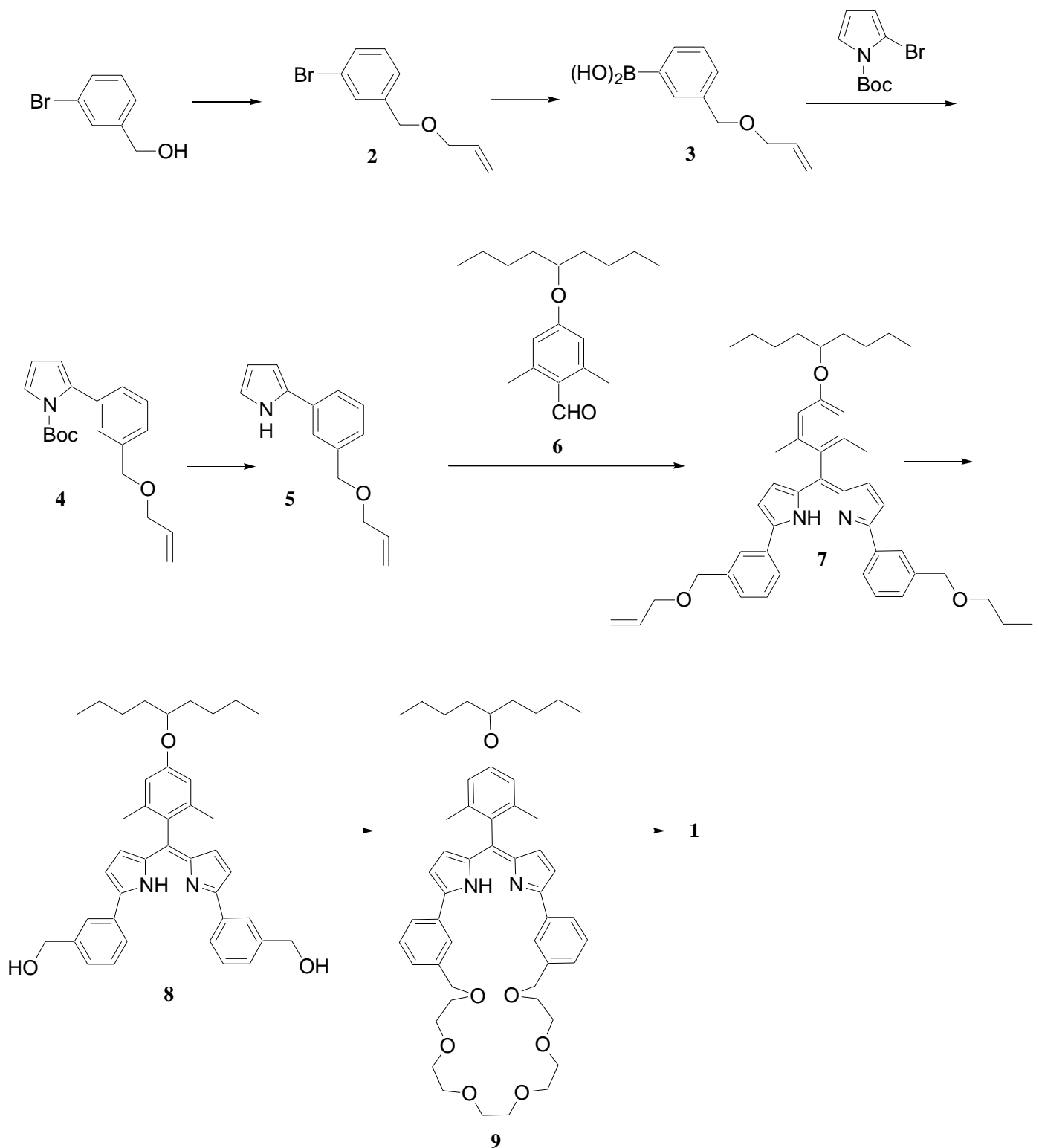

1-Allyloxymethyl-3-bromobenzene (2). Allyl bromide $(25.0 \mathrm{~mL}, 286 \mathrm{mmol})$ and 3-bromobenzylalcohol (50.0 g, $267 \mathrm{mmol})$ were dissolved in dry THF (400 mL). To a suspension of sodium hydride ( $21.2 \mathrm{~g}$ (60\% in oil), $534 \mathrm{mmol})$, previously washed three times with dry hexane in dry THF (200 mL) was added dropwise to the solution in an ice-water bath under a nitrogen atmosphere, and the reaction mixture was stirred for $3 \mathrm{~h}$ at room temperature. After quenching with methanol, the solvent was evaporated under reduced pressure. The mixture was neutralized with dilute hydrochloric acid, and the aqueous layer was extracted three times with ethyl acetate. The combined organic layer was washed with brine, dried over sodium sulfate, and evaporated. The residue was purified by 
column chromatography ( silica gel, hexane : ethyl acetate $=100: 1$ ) to give a colorless viscous liquid (59.8 g, 98\%). $\mathrm{R}_{\mathrm{f}}: 0.15$ (silica gel, hexane : ethyl acetate $\left.=100: 1\right) .{ }^{1} \mathrm{H} \mathrm{NMR}\left(300 \mathrm{MHz}, \mathrm{CDCl}_{3}\right) \delta 7.51$ $(\mathrm{s}, 1 \mathrm{H}), 7.41(\mathrm{~d}, J=8 \mathrm{~Hz}, 1 \mathrm{H}), 7.33-7.23(\mathrm{~m}, 1 \mathrm{H}), 7.22(\mathrm{dd}, J=8,8 \mathrm{~Hz}, 1 \mathrm{H}), 5.95(\mathrm{ddt}, J=17,11,6 \mathrm{~Hz}$, 1H), 5.32 (ddt, $J=17,2,1 \mathrm{~Hz}, 1 \mathrm{H}), 4.48$ (s, 2H), 4.03 (ddd, $J=6,1,1 \mathrm{~Hz}, 1 \mathrm{H})$.

3-(Allyloxymetyl)phenylboronic acid (3). A solution of 2 (33.2 g, $146 \mathrm{mmol})$ in dry THF (50 mL) was added dropwise with stirring for $3 \mathrm{~h}$ to a solution of $1.6 \mathrm{M} n$-butyllithium in hexane $(100 \mathrm{~mL})$ diluted with dry THF $(150 \mathrm{~mL})$ in a dry ice-acetone bath under a nitrogen atmosphere. After the mixture was stirred for an additional hour, a solution of trimethylborate $(26.8 \mathrm{~mL}, 240 \mathrm{mmol})$ in THF $(50 \mathrm{~mL})$ was added dropwise at $-78^{\circ} \mathrm{C}$. The reaction mixture was allowed to warm to room temperature and stirred for $20 \mathrm{~h}$. Dilute hydrochloric acid (0.1 M) was slowly added, and stirred over night. The organic layer was separated, and dried over sodium sulfate. Evaporation of the solvent afforded a crude viscous liquid (32.0 g), which was used in the next step without further purification.

N-tert-Butoxycarbonyl-2-(3-allyloxymethyl)phenylpyrrole (4). A suspension of the crude oil 3 $\left.\begin{array}{lllllll}15.7 & \mathrm{~g}\end{array}\right), \quad$ N-tert-butoxycarbonyl-2-bromopyrrole ${ }^{4} \quad(14.5 \quad \mathrm{~g}, \quad 63.8 \quad \mathrm{mmol})$, tetrakis(triphenylphosphine)palladium(0) (0.67 g, $1.73 \mathrm{mmol})$, toluene (200 mL), methanol (40 mL), and $2.0 \mathrm{M} \mathrm{Na}_{2} \mathrm{CO}_{3}$ aqueous solution $(56 \mathrm{~mL}, 112 \mathrm{mmol})$ were degassed and backfilled with nitrogen four times, and then stirred at $80^{\circ} \mathrm{C}$ for $20 \mathrm{~h}$. After cooling, the organic layer was separated, washed with brine, and dried over sodium sulfate. After concentration, the residue was purified by column chromatography ( g, $62 \%$ (total yield from 2)). $\mathrm{R}_{\mathrm{f}}$ : 0.10 (silica gel, toluene : hexane = $\left.1: 1\right) .{ }^{1} \mathrm{H} \mathrm{NMR}\left(300 \mathrm{MHz}, \mathrm{CDCl}_{3}\right)$

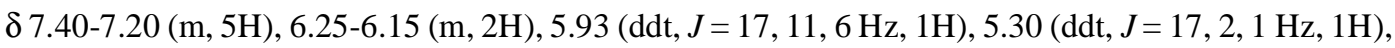
$5.20(\mathrm{ddt}, J=11,2,1 \mathrm{~Hz}, 1 \mathrm{H}), 4.54(\mathrm{~s}, 2 \mathrm{H}), 4.05(\mathrm{ddd}, J=6,1,1 \mathrm{~Hz}, 2 \mathrm{H}), 1.35$ (s, 9H).

2-(3-Allyloxymethyl)phenylpyrrole (5). A methanol solution of sodium methoxide (28\%, $25.0 \mathrm{ml})$ was added to a stirred solution of $4(7.20 \mathrm{~g}, 23.0 \mathrm{mmol})$ in dry THF $(150 \mathrm{~mL})$ and stirred at room temperature for $40 \mathrm{~min}$. The solution was diluted with toluene, and the organic layer was washed with deionized water (three times) and brine, and then dried over sodium sulfate. Evaporation of the solvent afforded a crude viscous liquid $(4.74 \mathrm{~g}, 22.2 \mathrm{mmol}, 97 \%)$, which was used in the next step without further purification.

\section{4-(1-Butylpentyloxy)-2,6-dimethylbenzaldehyde (6). A $\quad$ suspension of} 2,6-dimethyl-4-hydroxybenzaldehyde ${ }^{5}$ (39.0 g, $\left.260 \mathrm{mmol}\right), \mathrm{K}_{2} \mathrm{CO}_{3}$ (102 g, $738 \mathrm{mmol}$ ), and 5-nonyl methanesulfonate $^{6}(45.5 \mathrm{~g}, 205 \mathrm{mmol})$, which was prepared from 5-nonanol and mesyl chloride, in acetone $(500 \mathrm{~mL})$ was refluxed for $36 \mathrm{~h}$. After evaporation, the residue was washed with hexane and 
the filtrate was concentrated under vacuum. The residue was purified by column chromatography (silica gel, hexane : ethyl acetate $=50: 1$ ) to afford a yellow liquid $(28.2 \mathrm{~g}, 50 \%) . \mathrm{R}_{\mathrm{f}}: 0.15$ (silica gel, hexane : ethyl acetate $=50: 1) .{ }^{1} \mathrm{H} \mathrm{NMR}\left(300 \mathrm{MHz}, \mathrm{CDCl}_{3}\right) \delta 10.46(\mathrm{~s}, 1 \mathrm{H}), 6.55(\mathrm{~s}, 2 \mathrm{H}), 4.32$ (quintet, $J=6 \mathrm{~Hz}, 1 \mathrm{H}), 2.60(\mathrm{~s}, 6 \mathrm{H}), 1.79-1.19(\mathrm{~m}, 12 \mathrm{H}), 0.90(\mathrm{t}, J=7 \mathrm{~Hz}, 6 \mathrm{H})$.

\section{5-[4-(1-Butylpentyloxy)-2,6-dimethylphenyl]-1,9-bis[3-(allyloxymethyl)phenyl]-10H-dipyrrin}

(7). The aryl pyrrole $5(4.90 \mathrm{~g}, 23.0 \mathrm{mmol})$ and the benzaldehyde $6(3.17 \mathrm{~g}, 11.5 \mathrm{mmol})$ were dissolved in $\mathrm{CH}_{2} \mathrm{Cl}_{2}(900 \mathrm{~mL})$ and the solution was degassed with nitrogen for $1 \mathrm{~h}$. Trifluoroacetic acid $(0.50 \mathrm{~mL}$, $6.5 \mathrm{mmol}$ ) was added, and the solution was stirred for $21 \mathrm{~h}$ at room temperature under a nitrogen atmosphere. Then, 2,3-dichloro-5,6-dicyano-1,4-benzoquinone (2.61 g, $11.5 \mathrm{mmol})$ was added, and the solution was stirred for a further $2 \mathrm{~h}$. The purple solution was washed with $\mathrm{NaHCO}_{3}$ aq. and brine, dried over sodium sulfate, and evaporated. The residue was purified by column chromatography (aluminium oxide, hexane : methylene chloride $=3: 1)$ to give a red-purple viscous liquid $(3.35 \mathrm{~g}$, $43 \%)$. $\mathrm{R}_{\mathrm{f}}: 0.10$ (aluminium oxide, hexane : methylene chloride $\left.=3: 1\right) .{ }^{1} \mathrm{H} \mathrm{NMR}\left(300 \mathrm{MHz}, \mathrm{CDCl}_{3}\right) \delta$ $7.91(\mathrm{~s}, 2 \mathrm{H}), 7.59-7.32(\mathrm{~m}, 6 \mathrm{H}), 6.80(\mathrm{~d}, J=4 \mathrm{~Hz}, 2 \mathrm{H}), 6.65(\mathrm{~s}, 2 \mathrm{H}), 6.53(\mathrm{~d}, J=4 \mathrm{~Hz}, 2 \mathrm{H}), 5.97$ (ddt, $J=17,10,6 \mathrm{~Hz}, 2 \mathrm{H}), 5.33(\mathrm{ddt}, J=17,2,1 \mathrm{~Hz}, 2 \mathrm{H}), 5.21(\mathrm{ddt}, J=10,2,1 \mathrm{~Hz}, 2 \mathrm{H}), 4.64(\mathrm{~s}, 4 \mathrm{H}), 4.32$ (quintet, $J=6 \mathrm{~Hz}, 1 \mathrm{H}), 4.08$ (ddd, $J=6,1,1 \mathrm{~Hz}, 4 \mathrm{H}), 2.16$ (s, 6H), 1.90-1.29 (m, 12H), 0.94 (t, $J=7$ $\mathrm{Hz}, 6 \mathrm{H})$.

\section{5-[4-(1-Butylpentyloxy)-2,6-dimethylphenyl]-1,9-bis[3-(hydroxymethyl)phenyl]-10H-dipyrrin}

(8). A suspension of 7 (499 mg, $0.73 \mathrm{mmol}), 10 \% \mathrm{Pd} / \mathrm{C}(50 \mathrm{mg}$ ), $p$-toluenesulfonic acid monohydrate (50 mg, $0.26 \mathrm{mmol})$, and $\mathrm{H}_{2} \mathrm{O}(10 \mathrm{~mL})$ in methanol $(50 \mathrm{~mL})$ was refluxed for $24 \mathrm{~h}$. The reaction mixture was filtered through celite to remove the suspended $\mathrm{Pd} / \mathrm{C}$, and the filtrate was evaporated. The residue was purified by preparative TLC (silica gel, toluene : ethyl acetate $=2: 1$ ) to give a red solid (168 mg, 38\%). $\mathrm{R}_{\mathrm{f}}: 0.35$ (silica gel, toluene : ethyl acetate $\left.=2: 1\right) .{ }^{1} \mathrm{H}$ NMR (300 MHz, $\left.\mathrm{CDCl}_{3}\right) \delta 8.11$ $(\mathrm{s}, 2 \mathrm{H}), 7.78(\mathrm{~d}, J=8 \mathrm{~Hz}, 2 \mathrm{H}), 7.45(\mathrm{dd}, J=8,8 \mathrm{~Hz}, 2 \mathrm{H}), 7.32(\mathrm{~d}, J=8 \mathrm{~Hz}, 2 \mathrm{H}), 6.80(\mathrm{~d}, J=4 \mathrm{~Hz}, 2 \mathrm{H})$, $6.65(\mathrm{~s}, 2 \mathrm{H}), 6.53(\mathrm{~d}, J=4 \mathrm{~Hz}, 2 \mathrm{H}), 4.80(\mathrm{~s}, 4 \mathrm{H}), 4.27$ (quintet, $J=6 \mathrm{~Hz}, 1 \mathrm{H}$ ), $2.16(\mathrm{~s}, 6 \mathrm{H}), 1.90-1.29$ (m, 12H), $0.94(\mathrm{t}, J=7 \mathrm{~Hz}, 6 \mathrm{H})$.

Bridged dipyrromethene (9). Sodium hydride (100 $\mathrm{mg}$ (60\% in oil), $2.50 \mathrm{mmol}$, previously washed three times with dry hexane) was added to a suspension of $8(82.2 \mathrm{mg}, 0.136 \mathrm{mmol})$, pentaethylene glycol dimesylate ${ }^{7}(53.8 \mathrm{mg}, 0.136 \mathrm{mmol})$, and $\mathrm{K}_{2} \mathrm{CO}_{3}(200 \mathrm{mg}, 1.45 \mathrm{mmol})$ in $20 \%$ DMF/THF $(150 \mathrm{~mL})$, and then the mixture was stirred at $75^{\circ} \mathrm{C}$ for 5 days under a nitrogen atmosphere. After quenching with methanol, the mixture was diluted with toluene. The organic layer was then washed with water (three times) and brine, dried over sodium sulfate, and evaporated. The residue was purified by preparative TLC (silica gel, toluene : ethyl acetate $=1: 5$ ) to give a red viscous liquid (32.7 
mg, 30\%). $\mathrm{R}_{\mathrm{f}}: 0.28$ (silica gel, toluene : ethyl acetate $\left.=1: 5\right) .{ }^{1} \mathrm{H} \mathrm{NMR}\left(300 \mathrm{MHz}, \mathrm{CDCl}_{3}\right) \delta 7.95(\mathrm{~s}$, 2H), $7.87(\mathrm{~d}, J=8 \mathrm{~Hz}, 2 \mathrm{H}), 7.53(\mathrm{t}, J=8 \mathrm{~Hz}, 2 \mathrm{H}), 7.44(\mathrm{~d}, J=8 \mathrm{~Hz}, 2 \mathrm{H}), 6.79$ (d, $J=4 \mathrm{~Hz}, 2 \mathrm{H}), 6.65$ (s, 2H), $6.53(\mathrm{~d}, J=4 \mathrm{~Hz}, 2 \mathrm{H}), 4.72(\mathrm{~s}, 4 \mathrm{H}), 4.27$ (quintet, $J=7 \mathrm{~Hz}, 1 \mathrm{H}), 3.74-3.52(\mathrm{~m}, 20 \mathrm{H}), 2.16$ (s, $6 \mathrm{H}), 1.81-1.29(\mathrm{~m}, 12 \mathrm{H}), 0.94(\mathrm{t}, J=7 \mathrm{~Hz}, 6 \mathrm{H})$.

Target boron-dipyrromethene (1). Triethylamine $(0.60 \mathrm{~mL}, 4.3 \mathrm{mmol})$ was added to a solution of $9(14.2 \mathrm{mg}, 0.017 \mathrm{mmol})$ in toluene $(15 \mathrm{~mL})$. Boron trifluoride diethyl etherate $(0.90 \mathrm{~mL}, 7.1 \mathrm{mmol})$ was then added dropwise, and the solution was refluxed for $1 \mathrm{~h}$. After cooling, the reaction mixture was diluted with toluene, extracted with water (three times) and brine, dried over sodium sulfate, and concentrated. The residue was purified by preparative TLC ( silica gel, toluene : ethyl acetate $=1: 5$ ) to give a red-purple solid $(9.5 \mathrm{mg}, 63 \%)$. $\mathrm{R}_{\mathrm{f}}: 0.13$ (silica gel, toluene : ethyl acetate $\left.=1: 5\right)$. ${ }^{1} \mathrm{H}$ NMR (300 $\left.\mathrm{MHz}_{\mathrm{CDCl}}\right) \delta 7.90(\mathrm{~s}, 2 \mathrm{H}), 7.73(\mathrm{~d}, J=7 \mathrm{~Hz}, 2 \mathrm{H}), 7.45-7.38(\mathrm{~m}, 4 \mathrm{H}), 6.70(\mathrm{~d}, J=4 \mathrm{~Hz}, 2 \mathrm{H}), 6.67$ (s, $2 \mathrm{H}), 6.56(\mathrm{~d}, J=4 \mathrm{~Hz}, 2 \mathrm{H}), 4.64(\mathrm{~s}, 4 \mathrm{H}), 4.28$ (quintet, $J=7 \mathrm{~Hz}, 1 \mathrm{H}), 3.64-3.56(\mathrm{~m}, 20 \mathrm{H}), 2.20(\mathrm{~s}, 6 \mathrm{H})$, 1.81-1.30 (m, 12H), $0.94(\mathrm{t}, J=7 \mathrm{~Hz}, 6 \mathrm{H})$; ESI-MS: Calculated for $\mathrm{C}_{50} \mathrm{H}_{63} \mathrm{BF}_{2} \mathrm{~N}_{2} \mathrm{O}_{7}\left(\mathrm{MNa}^{+}\right): 875.46$; Found: 875.5. UV-vis: $\lambda_{\max }(\mathrm{nm}$, acetonitrile) (log $\varepsilon) 345$ (3.96), 552 (4.72). Fluorescence: $\lambda_{\max }(\mathrm{nm}$, acetonitrile) $\left(\boldsymbol{\Phi}_{f}\right) 589(0.68)$.

\section{Benesi-Hildebrand plot of 1 with $\mathrm{Na}^{+}$}

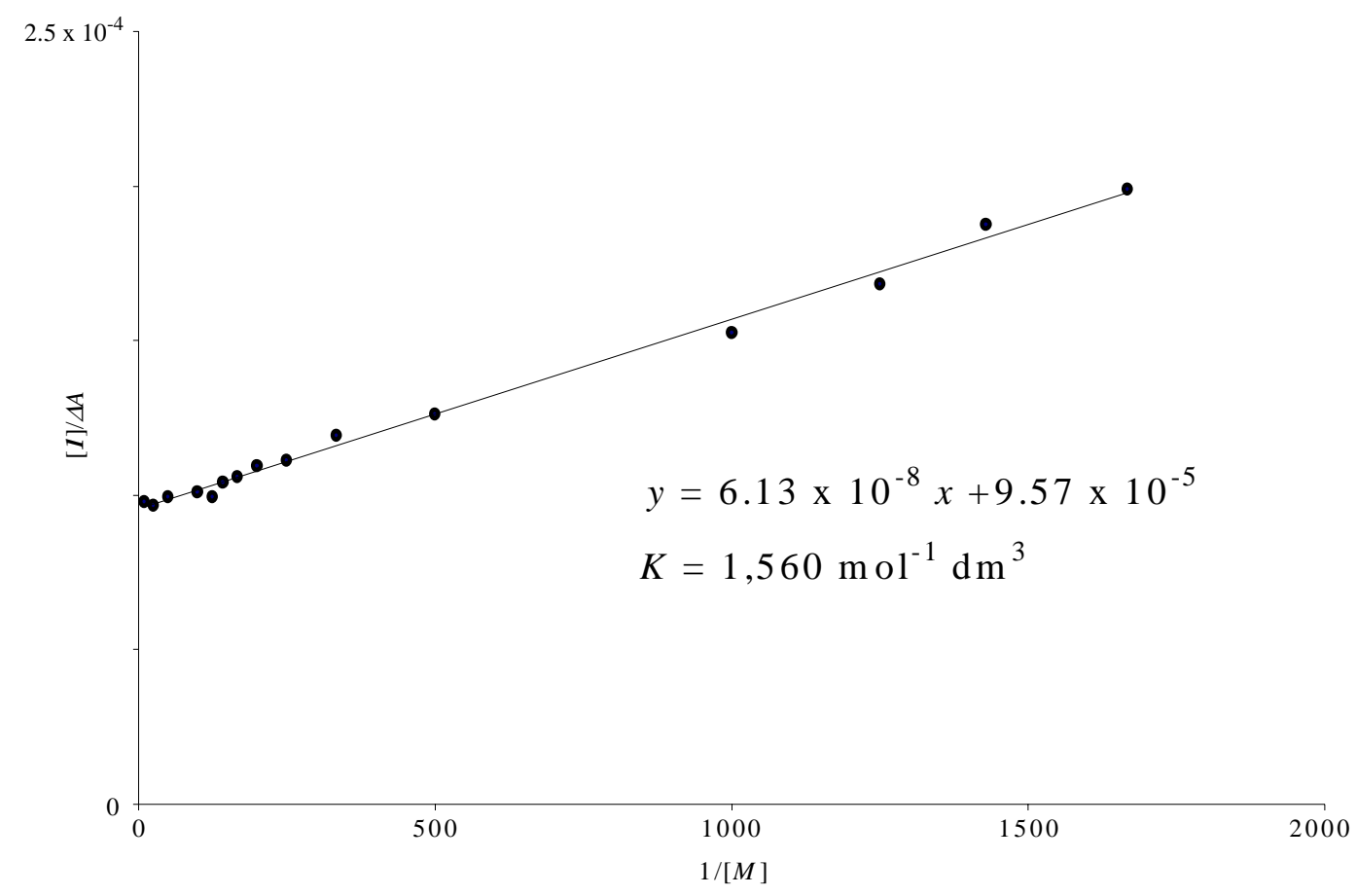




\section{Benesi-Hildebrand plot of 1 with $\mathrm{K}^{+}$}

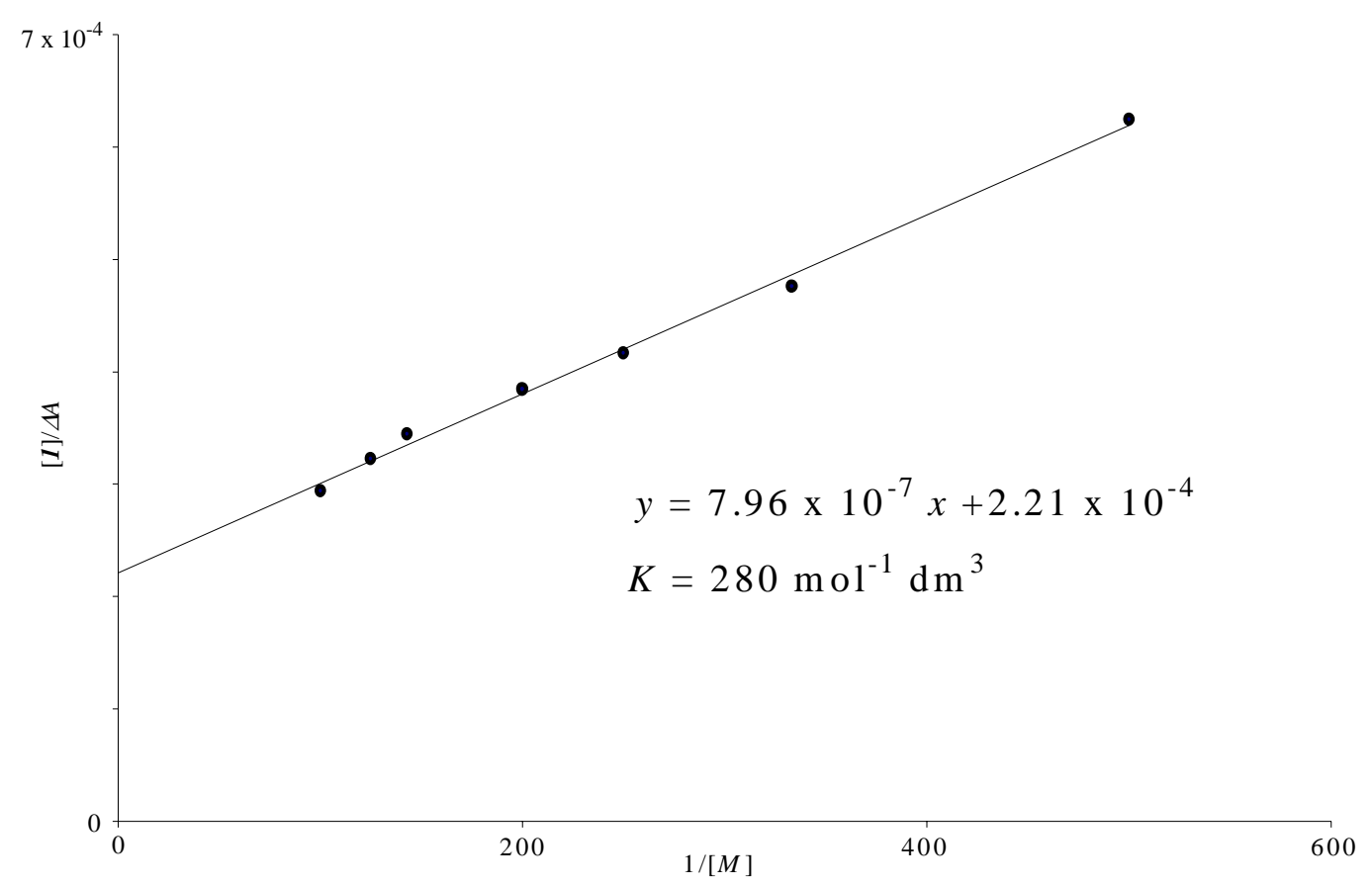

\section{References}

(1) Benesi, H. A.; Hildebrand, J. H. J. Am. Chem. Soc. 1949, 71, 2703-2707.

(2) Langhals, H.; Karolin, J.; Johansson, L. B. -^̊ J. Chem. Soc., Faraday Trans. 1998, 94, 2919-2922.

(3) Eaton, D. F. Pure Appl. Chem. 1988, 60, 1107-1114.

(4) Chen, W.; Stephenson, E. K.; Cava, M. P.; Jackson, Y. A. Org. Synth. 1991, 70, 151-156.

(5) Yamada, K.; Toyota, T.; Takakura, K.; Ishimaru, M.; Sugawara, T. New J. Chem. 2001, 25, 667-669.

(6) Foulon, V.; Asselberghs, S.; Geens, W.; Mannaerts, G. P.; Casteels, M.; Van Veldhoven. P. P. J. Lipid Res. 2003, 44, 2349-2355.

(7) Varshney, A.; Gray G. M. Inorg. Chem. 1991, 30, 1748-1754. 